\title{
MODELACIÓN DE LA DISTRIBUCIÓN GEOGRÁFICA POTENCIAL DE DOS ESPECIES DE PSITÁCIDOS NEOTROPICALES UTILIZANDO VARIABLES CLIMÁTICAS Y TOPOGRÁFICAS
}

\author{
Alexis Herminio PLASENCIA-VÁZQUEZ, ${ }^{1}$ Griselda ESCALONA- \\ SEGURA $^{1}$ Y LIGIA GUADALUPE ESPARZA-OLGUÍN ${ }^{1}$ \\ ${ }^{1}$ El Colegio de la Frontera Sur (ECOSUR), Unidad Campeche. Av. Rancho Polígono 2-A, Ciudad \\ Industrial de Lerma, C.P. 24500. San Francisco de Campeche, Campeche, México; alexpla79@gmail. \\ com, gescalon@ecosur.mx, esparzaligia@gmail.com
}

Plasencia-Vázquez, A. H., Escalona-Segura, G. y Esparza-Olguín, L. G. 2014. Modelación de la distribución geográfica potencial de dos especies de psitácidos neotropicales utilizando variables climáticas y topográficas. Acta Zoológica Mexicana (n.s.), 30(3): 471-490.

RESUMEN. Las variables climáticas y topográficas influyen en la selección del hábitat y en las tasas demográficas de muchas especies, por lo que son utilizadas para generar modelos de distribución geográfica potencial. En este estudio se identificó a las variables climáticas y topográficas más importantes en la generación de la distribución geográfica potencial del loro yucateco (Amazona xantholora) y el loro cabeza amarilla (Amazona oratrix) en la Península de Yucatán. Los modelos se generaron utilizando MaxEnt (algoritmo basado en la Máxima Entropía). Se emplearon los registros de presencia obtenidos de diferentes bases de datos disponibles de la Comisión Nacional de Áreas Naturales Protegidas (CONANP) y de El Colegio de la Frontera Sur (ECOSUR). Se realizaron 100 réplicas de los modelos por especie y se seleccionó como el mejor modelo el que representa el valor de la mediana. Para obtener un mapa de presencia/ausencia se aplicaron diferentes líneas de corte a este mejor modelo. Para validar el modelo se utilizaron registros de presencia y ausencia tomados en expediciones de campo posteriores. Para determinar las variables que contribuían más en la generación de los modelos se seleccionaron las de mayor aporte en el Jackknife de variables con los datos del AUC, el porcentaje de contribución de cada una y las curvas de respuesta. Amazona oratrix se distribuye potencialmente en la porción suroeste de la Península de Yucatán. Amazona xantholora está ausente en las áreas donde se localiza A. oratrix y se localiza en gran parte de la Península, incluyendo la Isla de Cozumel. La variable con mayor aporte al modelo de A. oratrix fue la temperatura promedio del trimestre más seco, mientras que para A. xantholora fueron el modelo digital de elevación y la precipitación de los trimestres más lluvioso y más frío. Para ninguna de las dos especies se observa una relación directa entre el gradiente de vegetación que se establece entre la porción norte y sur de la Península y el área de distribución geográfica potencial. El conocimiento particular sobre los factores ambientales que influyen en la distribución geográfica potencial de estas especies puede ser muy útil para acciones de conservación y para la evaluación futura de los cambios que estas puedan sufrir.

Recibido: 04/06/2013; aceptado: 11/04/2014 
Palabras clave: Factores abióticos, loros, modelos de distribución de especies, nicho ecológico, Psittacidae.

Plasencia-Vázquez, A. H., Escalona-Segura, G. \& Esparza-Olguín, L. G. 2014. Potential geographical distribution modeling of two neotropical parrot species using climatic and topographic variables. Acta Zoológica Mexicana (n.s.), 30(3): 471-490.

ABSTRACT. Topographic and climatic variables affect demographic rates and habitat selection of many species, so these variables are useful for generating potential distribution models. In this research, the most important topographic and climatic variables were identified for the generation of Yucatan parrot (Amazona xantholora) and Yellow-headed Parrot (Amazona oratrix) potential geographical distribution model in the Yucatan Peninsula. Models were generated using MaxEnt (algorithm based on Maximum Entropy). The presence records used were obtained from different available data bases from The National Commission of Natural Protected Areas (CONANP) and El Colegio de la Frontera Sur. One hundred replicas of models per species were developed and the best model selected is representing the median value. Different thresholds were applied to the best model in order to obtain a presence/ absence map. Presence and absence records taken from field work were used to validate the model. To know which of the variables contributed the most in the models generation, the ones with greater contribution in the variables Jackknife with the AUC data and because of the contribution percentage of each one, were selected. Amazona oratrix is potentially distributed in the southwestern portion of the Yucatan Peninsula. Amazona xantholora is present around much of the Peninsula, including the Cozumel Island, but it is absent within the areas where $A$. oratrix is located. The variable with greater contribution to $A$. oratrix model was the average temperature of the driest quarter, while for $A$. xantholora were the digital elevation model and the precipitation of the wettest and coldest quarters. For none of the two species it is observed a direct relationship between vegetation gradient established within the northern and southern of the Peninsula, and the potential geographical distribution area. The particular knowledge about the environmental factors that influence the potential geographical distribution of these species can be useful for conservation actions and for future evaluation of the changes that such distribution might suffer. Key words: Abiotic factors, ecological niche, species distribution models, parrots, Psittacidae.

\section{INTRODUCCIÓN}

Las variables climáticas y topográficas se han utilizado para generar modelos de distribución potencial (Marini et al. 2010, Kreakie et al. 2012) ya que se ha demostrado que la temperatura, la precipitación y la topografía afectan tanto la selección del hábitat como las tasas demográficas de especies (Davies et al. 2007, Green 2010). En este caso con el uso de variables climáticas, los algoritmos que estiman la distribución potencial de las especies con registros de solo presencia, identifican las áreas con características climáticas similares a los sitios en donde previamente han sido observadas la especies (Fischer et al. 2001). Se puede además incluir otros factores ambientales como la cubierta de vegetación y los usos de suelo (Contreras-Medina et al. 2010), los cuales son considerados como factores directos que determinan la presencia de las especies, pero se ha determinado que las variables climáticas y topográficas pueden ser variables condicionantes en la ocurrencia por sí solas (Davies et al. 2007, MotaVargas et al. 2013). Asimismo, en función del problema global del cambio climático 
que indica que los cambios en el clima (representado por variables como la temperatura y la precipitación) producen cambios en la distribución y abundancia de las especies de aves, se requiere hacer análisis de los efectos de las variables climáticas en los patrones de ocurrencia de las especies (Green 2010, Harris et al. 2014).

Los psitácidos son uno de los grupos de aves más amenazados en México, debido principalmente a la pérdida del hábitat y a la fuerte presión del tráfico de mascotas (Cantú-Guzmán et al. 2007). En México, se han realizado varios estudios para conocer la distribución potencial original y sus cambios en el tiempo por los usos de suelo para especies de loros, pericos y guacamayas utilizando el algoritmo genético para la producción de reglas (GARP, Genetic Algorithm for Rule Set Production) (Ríos-Muñoz \& Navarro-Sigüenza 2009, Monterrubio-Rico et al. 2010, Monterrubio-Rico et al. 2011). Estos estudios coinciden en que existe una pérdida del área de distribución de los psitácidos mexicanos. Sin embargo, el algoritmo GARP no tiene incorporado un procedimiento para la selección de las variables más importantes durante la generación de la distribución geográfica potencial de las especies (Elith 2000). Esta información permitiría reconocer los requerimientos ecológicos y ambientales de las especies de psitácidos, información que actualmente es aún muy limitada y específica para algunas regiones o para un número reducido de especies en áreas muy localizadas.

En un futuro cercano, los psitácidos también se pudieran ver afectados por los efectos de los cambios climáticos, que pudieran causar extinciones locales, variaciones en las áreas de distribución y recambio de especies (Peterson et al. 2002). Ante estas amenazas, es necesario conocer cuáles factores ambientales influyen más sobre la distribución de las diferentes especies, para poder determinar en cuáles regiones del país pudieran verse más afectadas.

Una zona importante para la conservación de los psitácidos en México es la Península de Yucatán, que alberga ocho de las especies presentes en el país (Mackinnon 2005). Entre estas especies está el loro cabeza amarilla Amazona oratrix, que está incluida en la NOM-059-2010 como en peligro de extinción (SEMARNAT 2010), y su situación es crítica por la pérdida del hábitat y por su elevado valor en el mercado ilegal de mascotas (Cantú-Guzmán et al. 2007). En Palizada, en el estado de Campeche, esta especie alcanza uno de los valores más elevados de abundancia relativa en todo México (Macías-Caballero \& Iñigo-Elías 2003). En la Península también está presente el loro yucateco Amazona xantholora, que es una especie endémica de la Provincia Biótica Península de Yucatán. Sin embargo, todavía se conoce poco a nivel regional sobre las variables ambientales que influyen en la distribución de estas especies. Por ello, el objetivo de este trabajo es identificar las variables climáticas y topográficas más importantes en la distribución geográfica potencial de dos especies de psitácidos en la Península de Yucatán, México mediante modelación con el algoritmo MaxEnt. 


\section{MATERIAL Y MÉTODOS}

El presente estudio generó la distribución geográfica potencial de dos de las especies de psitácidos que habitan en la Península de Yucatán, el loro yucateco (A. xantholora) y el loro cabeza amarilla (A. oratrix) (MacKinnon 2005). La distribución potencial se generó con los registros de presencia reportados en el Atlas de las aves de México (Navarro-Sigüenza et al. 2003), las bases de datos de la Comisión Nacional para la Conservación y Uso de la Biodiversidad (CONABIO) (http://www. conabio.gob.mx/), ORNISnet (http://www.ornisnet.org/) y los datos del Museo de la Biodiversidad Maya de la Universidad Autónoma de Campeche (CEDESU-UAC). Además, se incluyeron registros de los proyectos: Aviturismo en comunidades del estado de Campeche (2011), Monitoreo de aves durante la construcción, operación y mantenimiento de la línea de transmisión eléctrica Escárcega-Xpujil, en los Municipios de Escárcega y Calakmul, Campeche (2011), ambos liderados por El Colegio de la Frontera Sur (ECOSUR), y del plan eco-regional de las selvas Maya, Olmeca y Zoque 2003-2004; integración tri-nacional México-Guatemala-Belice; así como los registros de los censos realizados durante los años 2004 al 2009 en la Reserva de la Biosfera Ría Lagartos y los de Herrera-Herrera (2001), Gómez-Orduño (2006) y PucCabrera (2008).

La base de datos se constituyó por 147 registros de $A$. xantholora y nueve de $A$. oratrix, los cuales carecen de registros duplicados y estos fueron tomados entre los años de 1910 al 2009. Las capas climáticas que fueron utilizadas para generar los modelos cubrieron un intervalo temporal desde el año de 1910 hasta 2009, las cuales contaron con un mayor número de estaciones que las utilizadas por WorldClim y reúnen 19 variables bioclimáticas derivadas de valores mensuales de precipitación y temperatura (Cuervo-Robayo et al. 2013). Los datos de clima son ampliamente utilizados en la modelación de distribuciones geográficas potenciales (ej. Rojas-Soto et al. 2012, Mateo et al. 2013), ya que permiten utilizar un mayor número de registros históricos de las especies obtenidos en diferentes años sin incurrir en incongruencias temporales entre los registros y las coberturas utilizadas para modelar. De esta manera la idea es que se pueden sustituir otros tipos de coberturas como la vegetación, que pueden limitar los análisis ya que requieren de registros más actualizados de las especies que se correspondan solo al año en que fueron elaboradas.

Además, se utilizaron la altitud, la pendiente y el índice topográfico (http://gcmd. nasa.gov/records/GCMD_HYDRO1k.html) como variables predictoras indirectas. Para seleccionar estas variables se tuvo en cuenta que aunque la Península de Yucatán es un área con poca variación topográfica (Lugo-Hubp et al. 1992), la transición entre las zonas más húmedas y secas de la región ésta determinada por las dos unidades morfológicas principales que la componen.

Las capas fueron re-muestreadas a una resolución de 0,008333 (aproximadamente $1 \mathrm{~km}^{2}$ ) utilizando el programa ArcView 3.2 (ESRI 1999) y se recortaron para 
delimitar el área de estudio. Para reducir la co-linealidad entre las 19 variables bioclimáticas y las tres topográficas, se realizó un análisis de correlación y se seleccionaron aquellas que presentaron valores de $|\mathrm{r}|<0.7$ (Dormann et al. 2012). Entre las variables altamente correlacionadas se utilizaron aquellas con las cuales la relación con los patrones de distribución de las aves se explicaban mejor o que tuvieran una relación más directa con las especies en estudio (Fitzpatrick et al. 2013). Se tuvo en cuenta que la variabilidad climática, determinada por las fluctuaciones durante los meses extremos (mayor o menor precipitación, temperaturas más o menos elevadas), puede limitar la reproducción de los loros, ya sea por un incremento en la depredación de los nidos o por disminuciones en la disponibilidad de alimento (Renton \& Salinas-Melgoza 2004). El desarrollo fenológico de las plantas que utilizan los loros para alimentarse depende de la lluvia, la temperatura y la radiación solar, que son los reguladores principales en la floración y desarrollo de los frutos (Villers et al. 2009). A la par, las fluctuaciones espacio-temporales en la disponibilidad de alimentos durante todo el año provocan que los loros utilicen diferentes áreas, al realizar un seguimiento de los recursos alimenticios (Renton \& Salinas-Melgoza 2002, Renton 2009). Finalmente, se seleccionaron 11 variables ambientales para generar los modelos (Cuadro 1).

Los mapas de distribución potencial fueron generados en el programa MaxEnt (Phillips et al. 2006) (http://www.cs.princeton.edu/ schapire/maxent/), considerado como uno de los de mejor desempeño (Elith \& Graham 2006). Además, se ha comprobado que con este programa se pueden obtener resultados relativamente adecua-

Cuadro 1. Variables climáticas (Cuervo-Robayo et al. 2013) y topográficas (proyecto Hydro 1k) empleadas para la modelación de la distribución potencial de Amazona xantholora y Amazona oratrix en la Península de Yucatán.

\begin{tabular}{lc}
\hline Variables climáticas & Abreviaturas \\
\hline Temperatura máxima promedio del periodo más cálido $\left({ }^{\circ} \mathrm{C}\right)$ & Bio5 \\
Temperatura promedio del trimestre más seco $\left({ }^{\circ} \mathrm{C}\right)$ & Bio9 \\
Temperatura promedio del trimestre más cálido $\left({ }^{\circ} \mathrm{C}\right)$ & Bio10 \\
Temperatura promedio del trimestre más frío $\left({ }^{\circ} \mathrm{C}\right)$ & Bio11 \\
Precipitación del trimestre más lluvioso $(\mathrm{mm})$ & Bio16 \\
Precipitación del trimestre más seco $(\mathrm{mm})$ & Bio17 \\
Precipitación del trimestre más cálido $(\mathrm{mm})$ & Bio18 \\
Precipitación del trimestre más frío $(\mathrm{mm})$ & Bio19 \\
Variables topográficas & \\
Modelo digital de elevación & H_DEMP \\
Pendiente & H_SLOPE \\
Índice topográfico (humedad) & H_TOPOIND \\
\hline
\end{tabular}


dos con tamaños de muestra pequeños (p.ej. Hernandez et al. 2006, Pérez-García \& Liria 2013). Durante la modelación se mantuvieron los parámetros predeterminados por el programa MaxEnt, como ha sido sugerido por Phillips et al. (2006) y validado en algunos estudios (p.ej. Phillips \& Dudík 2008). Se realizaron 100 réplicas para cada modelo utilizando como método de remuestreo el bootstrap. El 30\% de los registros históricos de A. xantholora se utilizó para la validación del modelo y el 70\% restante para generar el modelo. En el caso de $A$. oratrix como se tenían pocos registros históricos todos se usaron para correr el modelo.

Con los valores del AUC de las 100 réplicas de los modelos de cada especie se construyeron histogramas de frecuencias y de esta manera se observó una tendencia general de los datos hacia una distribución normal. Para comprobar estadísticamente esta tendencia se aplicó un test de Shapiro-Wilk con el programa Statistica 8.0. Para ambas especies se comprobó que los valores del AUC para los 100 modelos tienen una distribución normal: A. oratrix $(\mathrm{W}=0,98 ; \mathrm{p}=0.12)$ y A. xantholora $(\mathrm{W}=0.98$; $\mathrm{p}=0.09$ ). Dado este comportamiento, entre las 100 réplicas del modelo se seleccionó como el mejor el que representa el valor de la mediana, para evitar el efecto de los valores extremos. Debido a que el valor de la media coincide con la mediana para ambas especies, cualquiera de las dos medidas sería útil. Para obtener un mapa de presencia/ausencia se aplicaron diferentes líneas de corte a este mejor modelo. Estas se establecieron teniendo en cuenta cuatro de los algoritmos más utilizados y recomendados en la literatura: el de presencia mínima de los puntos de entrenamiento (minimum training presence, MTP), el 10 percentil (10 percentile training presence, 10P), la sensibilidad y especificidad equivalentes (equal training sensitivity and specificity, OC) y la de sensibilidad máxima de entrenamiento más especificidad (Maximum training sensitivity plus specificity, Max) (Liu et al. 2005).

Para determinar la línea de corte con que se obtenía el mejor mapa binario se utilizó una matriz de confusión con los registros obtenidos para validación en campo, que es un método que se utiliza para medir el desempeño de los modelos (Fielding \& Bell 1997). Con esta matriz se determinó la coincidencia entre los registros de validación y la predicción que hace el modelo sobre el espacio geográfico. De esta forma se comprueba si hay sobreestimación o una elevada omisión en los modelos obtenidos. Se comprobó cuántos de los registros de presencia y ausencia eran predicciones correctas. Además, se determinó la cantidad de falsos negativos (registros de presencia predichos como ausencias) y los falsos positivos (registros de ausencia predichos como presencia).

Para ello se utilizaron registros de presencia y ausencia de las especies, independientes de los utilizados para obtener los modelos, tomados en 12 expediciones de campo realizadas durante los años 2011 y 2012, en diferentes áreas de la Península. Las áreas seleccionadas para la validación en campo incluían sitios donde los modelos predecían ausencia y presencia de las especies. Además, se tuvieron en cuenta 
registros del monitoreo realizado en el año 2010 en la Reserva de la Biosfera Ría Lagartos. En cada área, se tomaron registros estableciendo10 puntos de conteo de radio ilimitado (Hutto et al. 1986) que se recorrían al amanecer entre la 05:30 y las 10:00, ya que en ese horario se incrementan los niveles de actividad de los loros neotropicales (Salinas-Melgoza \& Renton 2005). También se tomaron registros dos horas antes de la puesta de sol, que es cuando se da un segundo pico de actividad de los loros; para ello se siguió el método de itinerario de censo según Blondel (1969). En total, para la validación en campo se utilizaron 149 registros de presencia y 50 registros de ausencia para $A$. xantholora y 50 y 150 registros respectivamente para $A$. oratrix. Para $A$. oratrix los registros obtenidos en el trabajo de campo superaron a los registros históricos.

Para evaluar la contribución de las variables ambientales a la predicción de los modelos de cada especie se analizaron los resultados de dos vías que el programa MaxEnt utiliza: la estimación de los porcentajes de contribución relativa al modelo y los resultados de la prueba Jackknife. Se utilizaron los valores de resumen de los resultados de los 100 modelos, que contienen los valores de la media para todos los parámetros analizados.

En el caso de la prueba de Jackknife se crean tres tipos de modelos. El primer tipo de modelo se construye con todas las variables excluyendo una variable ambiental, para determinar así como la variable excluida afecta al modelo. El siguiente modelo se crea usando cada variable ambiental de manera independiente, para observar cómo es su aporte individual al modelo general. Por último se construye un modelo usando todas las variables. Entre los modelos creados con las variables ambientales independientes, se seleccionó como la variable más importante aquella que tuviera un mayor aporte al modelo general. Entre los modelos creados excluyendo una de las variables ambientales, se seleccionó como la variable más importante aquella que al ser excluida provoque que el aporte de las demás variables al modelo sea menor.

Para determinar la contribución relativa (\%) de cada variable ambiental al modelo, en cada iteración del algoritmo de entrenamiento, se añade el aumento en la ganancia regularizada a la contribución de la variable correspondiente (Phillips et al. 2006). Al final del proceso de entrenamiento del modelo los valores de contribución de cada variable ambiental se convierten a porcentajes. Entre las diferentes variables ambientales, se seleccionaron como las de mayor aporte a los modelos aquellas cuya contribución relativa fuera mayor del 50\%. En el caso de que ninguna variable cumpliera con esta condición, se seleccionaron como las más importantes las primeras que tuvieran los mayores porcentajes de contribución y que sumados alcanzaran más del 50\%.

Para poder visualizar cómo es que las variables ambientales con mayor aporte influyen sobre la predicción del modelo se utilizaron las curvas de respuesta. Estas curvas muestran cómo afecta cada variable ambiental a la predicción de MaxEnt, te- 
niendo en cuenta la probabilidad predicha de condiciones idóneas frente a los valores de cada variable ambiental. Se generan dos tipos de curvas, una en la que se toma una variable ambiental y se va variando su valor mientras las demás se mantienen constantes. Este método es efectivo cuando las variables ambientales son independientes unas de otras. Cuando las variables ambientales están fuertemente correlacionadas MaxEnt genera un segundo conjunto de curvas donde el modelo se construye solamente con la variable correspondiente (Phillips et al. 2006).

\section{RESULTADOS}

El valor del AUC del mejor modelo de distribución geográfica potencial de $A$. xantholora, con los datos de entrenamiento, fue de 0.92; mientras que el de validación fue de 0.83. En el caso de A. oratrix, el valor del AUC fue de 0.99 con los datos de entrenamiento. Los modelos predicen con alta probabilidad la distribución de ambas especies. En relación a las líneas de corte, para A. oratrix coinciden los resultados para los cuatro algoritmos, mientras que para $A$. xantholora solo coinciden los valores en los algoritmos OC y Max (Cuadro 2).

Para A. xantholora los mejores resultados se obtuvieron con el algoritmo MTP, ya que con los demás se subestima el área de distribución geográfica potencial de la especie y existe una menor coincidencia entre los registros de validación en campo y los resultados del modelo (Cuadro 3). Con A. oratrix, el 94\% de los registros de presencia tomados en campo coinciden con la predicción del modelo, mientras que los de ausencia coinciden en un $100 \%$. Amazona oratrix se distribuye potencialmente en la porción suroeste de la Península de Yucatán (Fig. 1A). Amazona xantholora está ausente en las áreas donde se localiza $A$. oratrix y se localiza en gran parte de la Península, incluyendo la Isla de Cozumel (Fig. 1B).

Para ambas especies, las variables con los mayores porcentajes de aporte a los modelos y las de mayor contribución por la prueba de Jackknife coincidieron (Cuadro 4, Fig. 2). La variable ambiental con mayor aporte al modelo de A. oratrix fue la temperatura promedio del trimestre más seco, mientras que para $A$. xantholora fueron

Cuadro 2. Valores de las diferentes líneas de corte evaluadas con cuatro algoritmos para obtener los modelos de distribución geográfica potencial de las especies Amazona oratrix y Amazona xantholora en la Península de Yucatán.

\begin{tabular}{ccc}
\hline Algoritmo & Amazona xantholora & Amazona oratrix \\
\hline MTP & 0.10 & 0.43 \\
10P & 0.34 & 0.43 \\
OC & 0.41 & 0.43 \\
Max & 0.41 & 0.43 \\
\hline
\end{tabular}


Cuadro 3. Matriz de confusión para las distintas líneas de corte establecidas con diferentes algoritmos para obtener los mapas de presencia/ausencia de Amazona xantholora en la Península de Yucatán.

\begin{tabular}{cccc}
\hline \multicolumn{2}{c}{ Amazona xantholora } & \multicolumn{2}{c}{ Predicción del modelo } \\
\hline Datos de campo & Algoritmo & Ausencia & Presencia \\
\hline Puntos de Ausencias $(\mathrm{n}=50)$ & OC & 50 & 0 \\
& MTP & 50 & 0 \\
& Max & 50 & 0 \\
Puntos de Presencias (n=149) & 10P & 50 & 0 \\
& OC & 50 & 99 \\
& MTP & 3 & 146 \\
& Max & 50 & 99 \\
& 10P & 44 & 105 \\
\hline
\end{tabular}

el modelo digital de elevación y la precipitación de los trimestres más lluvioso y más frío. La distribución geográfica potencial de A. oratrix parece estar mejor condicionada por variables de temperatura, mientras que la de $A$. xantholora por variables de precipitación y topográficas. El comportamiento de las variables ambientales es semejante para los dos tipos de curvas (Fig. 3), lo cual demuestra que el proceso de eliminación de las variables correlacionadas fue efectivo y se logró la independencia entre las variables.

En la Península de Yucatán, A. oratrix se distribuye con preferencia en áreas donde las temperaturas son más elevadas durante la temporada seca (Fig. 3). Por su parte, Amazona xantholora en la Península se localiza preferentemente en las áreas con una altitud superior a los $150 \mathrm{~m}$, en las cuales las precipitaciones durante el trimestre más

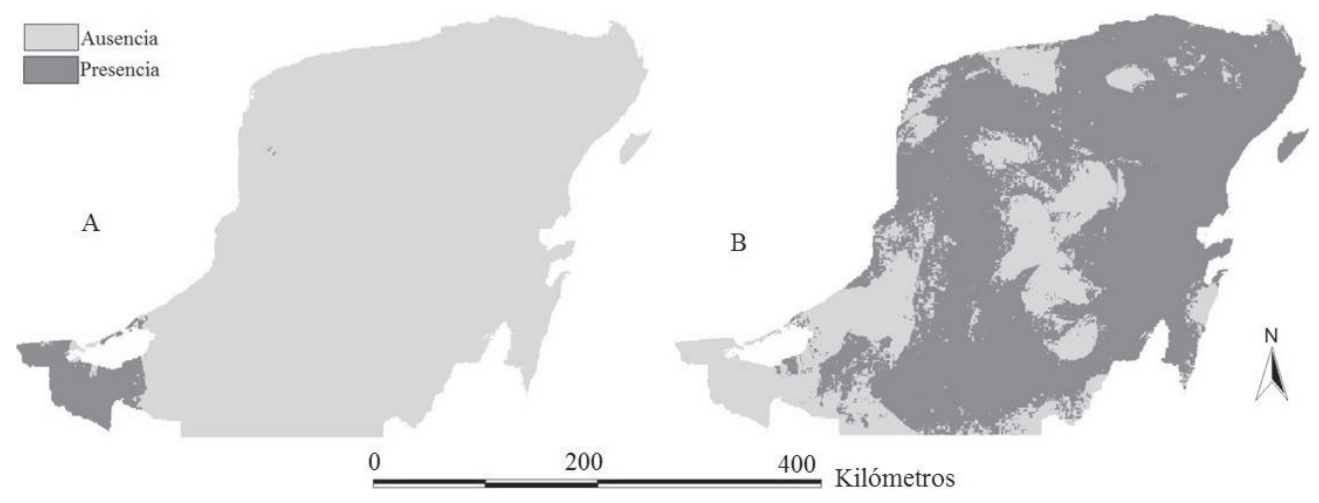

Figura 1. Distribuciones geográficas potenciales de A. oratrix (A) y A. xantholora (B) en la Península de Yucatán, México obtenidas con el algoritmo de Máxima Entropía (MaxEnt). 
Plasencia-Vázquez et al.: Distribución potencial de psitácidos neotropicales

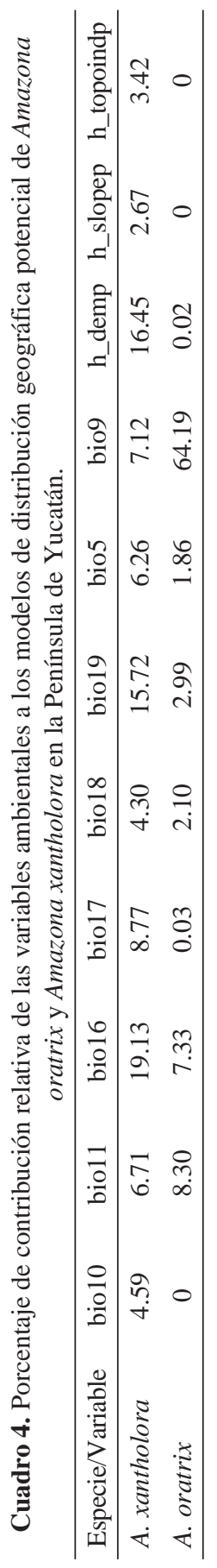




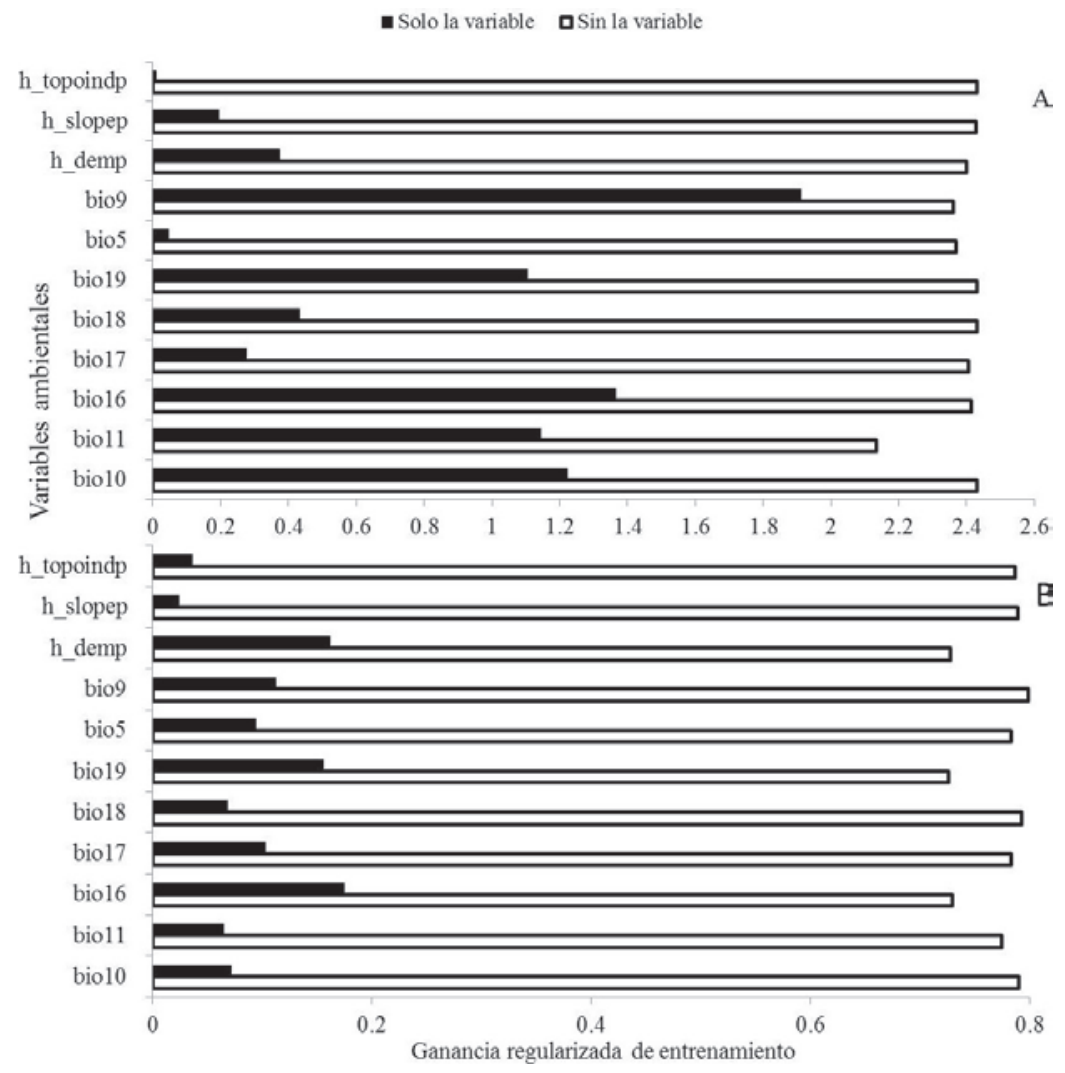

Figura 2. Resultado del Jackknife sobre la importancia de las variables considerando el área bajo la curva AUC con los datos de entrenamiento, en los modelos de distribución potencial de Amazona oratrix (A) y Amazona xantholora (B) en la Península de Yucatán, México.

lluvioso estén por debajo de los 600 mm y durante el trimestre más frío oscilen entre los 50-200 mm (Fig. 3).

\section{DISCUSIÓN}

Los modelos seleccionados para cada especie tuvieron un buen desempeño y se pueden considerar como robustos (Marmion et al. 2009). El uso de datos de solo presencia de las especies para obtener los modelos constituyó una ventaja, debido a que la obtención de datos reales de ausencia suele ser muy difícil y son menos probables de encontrar en las bases de datos con registros históricos (Galparsoro et al. 2009). Un problema serio para la modelación de la distribución de especies con modelos de solo presencia suelen ser los sesgos asociados al muestreo de los datos (Phillips et al. 
Plasencia-Vázquez et al.: Distribución potencial de psitácidos neotropicales
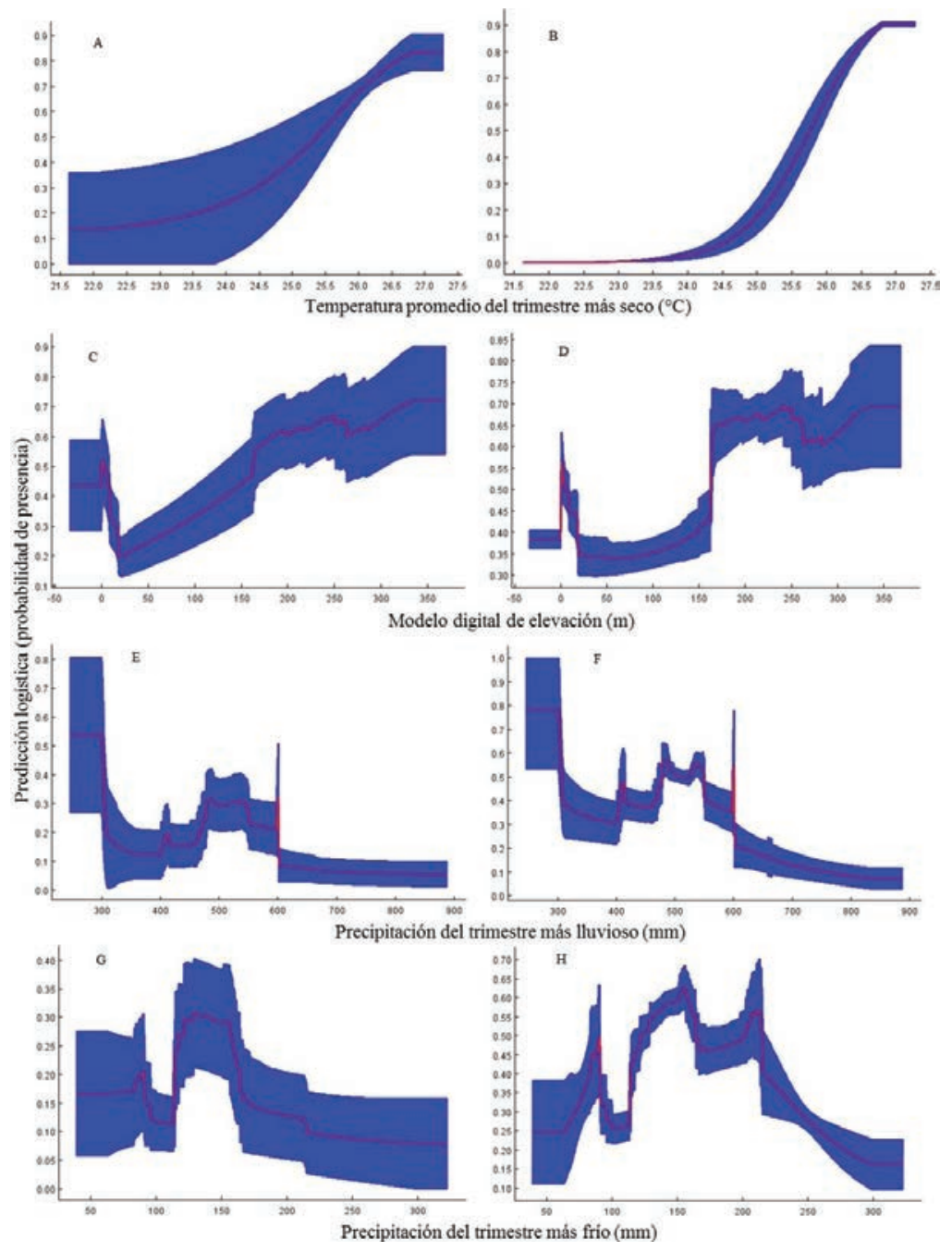

Figura 3. Curvas de respuesta de las variables ambientales utilizadas para generar los modelos de distribución geográfica potencial de Amazona oratrix (A-B) y Amazona xantholora (C-H) en la Península de Yucatán. Media de las 100 réplicas (rojo) +/- desviación estándar (azul). Columna de la izquierda teniendo en cuenta el efecto de todas las variables y columna de la derecha teniendo en cuenta solo el valor de la variable correspondiente.

2009). En este estudio se logró recopilar una cantidad de datos considerables de la especie A. xantholora, los cuales estaban bien distribuidos dentro del área de la Península, lo cual permitió generar un buen modelo. En el caso de A. oratrix, aún cuando los registros de presencia utilizados para modelar fueran pocos, de igual manera 
se obtuvo un modelo robusto, lo cual fue comprobado con los datos de validación tomados en campo. Se ha comprobado que el algoritmo MaxEnt funciona de manera adecuada para predecir zonas de presencia para especies para las que se cuenta con pocos registros (Pearson et al. 2007).

Otra fuente de error asociada a los modelos de solo presencia es el hecho de que al no contar con información confiable acerca de las ausencias la estimación de los errores de comisión se dificulta (Anderson et al. 2003). Aunque MaxEnt utiliza las pseudoausencias para la calibración del modelo, éstas normalmente no son válidas para su uso en la evaluación (Peterson et al. 2011). De ahí la importancia de utilizar ausencias verdaderas para validar los modelos y determinar que tanto coinciden las predicciones del modelo con lo que se observa en el campo.

El análisis del comportamiento de las réplicas de los modelos, según los valores del AUC, resultó ser una herramienta adecuada para la selección de los mejores. De esta manera se pudo distinguir cuál es la tendencia entre las réplicas y así determinar hacia donde apuntan la mayoría de los modelos, en función del tipo de distribución que sigan. La selección de los mejores modelos con MaxEnt es un punto crítico durante la modelación, ya que no se cuenta con un parámetro que de manera automática se pueda establecer para escoger el modelo más adecuado, como ocurre en el caso de otros algoritmos como GARP. En la literatura existen diversas vías que se han propuesto para la selección de los mejores modelos (ej. Baldwin 2009, Hoenes \& Bender 2010, Fitzpatrick et al. 2013), las cuales son producto de la constante evolución debido a las críticas y a la rapidez con que se desarrollan las nuevas tendencias en el ámbito de la modelación de nicho ecológico y los modelos de distribución geográfica potencial (Warren \& Seifert 2011, Royle et al. 2012, Yackulic et al. 2013). Pero a la fecha, es aún difícil justificar cuál de las metodologías propuestas es la más adecuada para seleccionar los mejores modelos con MaxEnt, ni bajo qué condiciones es más propicio usar una u otra, ya que en primera instancia depende de los objetivos particulares de cada investigación (Peterson et al. 2011).

Se comprobó que la distribución potencial de A. oratrix dentro de la Península de Yucatán está circunscrita a un área pequeña dentro del estado de Campeche. Se ha comprobado que los modelos realizados para especies de distribución restringida tienden a brindar mejores resultados que los desarrollados con especies de amplia distribución como A. xantholora (ej. Venier et al. 2004, Mateo et al. 2010). Aunque el área de presencia predicha para A. oratrix dentro de la Península de Yucatán coincide en su mayoría con la obtenida en los modelos realizados por Navarro \& Peterson (2007) y Ríos-Muñoz \& Navarro-Sigüenza (2009), nuestra modelación la amplió por los alrededores de la porción este de la Laguna de Términos, lo cual se comprobó efectivamente durante las expediciones de validación en campo.

En la porción suroeste de la Península de Yucatán, donde el modelo predice presencia de $A$. oratrix se encuentra ausente $A$. xantholora, lo cual se verificó durante las 
expediciones de validación en campo. El modelo ubica a $A$. xantholora en gran parte del territorio de la Península, que incluye las áreas más secas de la zona noroeste del estado de Yucatán (menos de 600 mm de precipitación como promedio anual) (Orellana-Lanza 2009) y algunas de las zonas más húmedas de la porción sur. Está ausente en algunas zonas con mayor pluviosidad de la región, entre ellas las pertenecientes a la sub-región Candelaria, que presenta valores muy altos de precipitación promedio anual, entre 1700 y 1800 mm (CONAGUA 2006).

La variabilidad climática que presenta la Península de Yucatán, que se refleja a través del gradiente de precipitación que va del sureste (áreas más húmedas) al noreste (áreas más secas) (White \& Darwin 1995), es una de las razones por las que se pueden realizar este tipo de estudios en la Península. Este gradiente es detectable por la transición de vegetación desde la selva baja en el norte a la selva alta en el sur (Orellana-Lanza 2009). Por ejemplo, en la región de Calakmul, Campeche se aprecia la transición de las zonas húmedas a las secas, particularmente de ambos lados de la meseta de Zoh-Laguna (18³5’24.54” N, 89²6’59.28” W) (Martínez \& GalindoLeal 2002). Para ninguna de las dos especies se observa una relación directa entre este gradiente de vegetación y el área de distribución geográfica potencial. Amazona xantholora se localiza potencialmente tanto en áreas de selva baja en la porción norte de la Península, como en áreas de selvas de tierras húmedas en el sur. Mientras que, A. oratrix solo se localiza en una pequeña porción de las áreas ocupadas por selvas más húmedas.

Con relación a las variables topográficas, a escala de país, la Península de Yucatán se ha descrito como una región de poco contraste en la altitud (Lugo-Hubp et al. 1992). Pero al hacer un análisis a escala de la Península, ésta se divide en dos unidades morfológicas principales: la primera está ubicada en el norte y en ella predominan las planicies; la segunda aparece en el sur donde las planicies alternan con lomeríos de hasta 400 m.s.n.m. Nuestros resultados indican que las características de las dos unidades morfológicas principales que componen la Península marcan una diferencia que puede afectar los patrones de distribución de los psitácidos en el área cuando se hacen estudios a escalas locales. Además, los valores más elevados de altitud dentro de la Península sólo alcanzan los 400 m.s.n.m. (Lugo-Hubp et al. 1992) y en general la mayoría de las especies de loros se han documentado por debajo de los 500 m.s.n.m. (Ríos-Muñoz 2002). Lo anterior hace de la Península un sitio con características apropiadas desde el punto de vista altitudinal para el desarrollo de este grupo.

Amazona oratrix tiene una distribución más restringida dentro de la Península y solo una variable de las que utilizamos para este trabajo tiene un aporte significativo en la construcción del modelo, ya que las condiciones ambientales bajo las cuales se desarrolla la especie están mejor definidas en la pequeña área que ocupa (Mateo et al. 2011). Para esta especie, la temperatura promedio en el trimestre más seco parece determinar su presencia en la región y en las zona donde el modelo predice su presencia 
se alcanzan los valores más elevados de temperatura dentro de la Península. Temperaturas superiores a $26.8^{\circ} \mathrm{C}$ se localizan hacia el Golfo de México, en el Estado de Campeche, siendo las más elevadas en Laguna de Términos y Ciudad del Carmen (Orellana-Lanza 2009) y justamente en los alrededores de estas áreas es donde se ha verificado la presencia de la especie.

Amazona oratrix se puede localizar en selva caducifolia y semicaducifolia, así como en bosque de ribera (Collar et al. 1992). Estas formaciones vegetales están presentes en otras áreas de la Península de Yucatán, en las cuáles no se tienen reportes de este loro ni se encontró durante los trabajos de validación en campo. Estas pruebas apoyan que $A$. oratrix requiere de hábitats muy cálidos para vivir, que solo encuentra en la porción suroeste de la Península. De igual manera, su presencia en esta área también puede estar determinada por otros factores de tipo ambiental o histórico que no se tuvieron en cuenta durante este estudio. Si se hubiese contado con un mayor número de registros históricos de la especie, de diferentes años, se hubiesen podido utilizar variables de vegetación para la obtención de los modelos. Además, se hubiesen podido integrar las variables de vegetación con variables que caracterizaran a factores tales como la degradación del hábitat, los usos del suelo y la captura para el mercado ilegal de psitácidos, las cuales afectan a las poblacionales de loros.

En el caso de $A$. xantholora sucede lo contrario a A. oratrix, y un mayor número de variables aportan de manera considerable al modelo. Esta especie ocupa potencialmente gran parte del territorio de la Península y entre las áreas que el modelo predice su ausencia están las pertenecientes a la sub-región Candelaria, que es la zona de mayor precipitación pluvial de la Península de Yucatán (CONAGUA 2006). La precipitación ha sido considerada como un factor crítico que regula la productividad de los ecosistemas (Svoray \& Karnieli 2010) y para esta especie la precipitación del trimestre más lluvioso y frío constituyen un factor limitante para su distribución en aquellas áreas donde alcanzan sus máximos valores.

Con otras especies del género Amazona se ha comprobado que las variaciones interanuales en la precipitación afectan el éxito reproductivo (Renton \& Salinas-Melgoza 2004). Amazona xantholora puede estar evitando las áreas de la Península con mayores valores de precipitación, en las cuales pueden darse mayores fluctuaciones interanuales (Márdero et al. 2012). La especie puede tener preferencia por aquellas áreas con niveles de pluviosidad más estables, donde no se den cambios bruscos en la abundancia o escasez de frutos, que pueden provocar fluctuaciones en las densidades y tasas de mortalidad de especies frugívoras (Wright et al. 1999, Renton \& SalinasMelgoza 2004). Además, la distribución potencial de A. xantholora en la Península se favorece en áreas que no superan los $300 \mathrm{~m}$ de altitud, lo cual concuerda con lo reportado para la mayor parte de los psitácidos mexicanos que están distribuidos en tierras bajas (Howell \& Webb 1995). Se ha comprobado que la altitud es uno de los factores más importantes que influyen en la distribución de los seres vivos, por lo 
que los patrones altitudinales de la diversidad están recibiendo cada vez más atención (Rahbek 2005).

La información particular generada para cada especie puede apoyar la planeación, ejecución y evaluación de acciones de conservación, ya que el conocimiento sobre los factores que influyen en la distribución de las especies es de importancia para los investigadores y administradores de la vida silvestre (Baldwin 2009). Se considera que la información generada en nuestro trabajo puede contribuir en la evaluación de los posibles cambios que pueden sufrir las áreas de distribución de estos psitácidos en la Península de Yucatán a partir de las modificaciones esperadas bajo condiciones de cambio climático, para las próximas décadas.

Se prevé en los próximos años una disminución de las lluvias anuales cerca de algunas áreas de la costa de la Península, que irán estabilizándose hacia el centro y con un aumento significativo de la precipitación hacia la zona occidental (Márdero et al. 2012). Estos cambios en las condiciones climáticas irán modificando el entorno de los hábitats de los loros en la Península, provocando cambios en la fenología de las especies vegetales de las cuales se alimentan. A mediano o largo plazo, las áreas de distribución de los psitácidos en la región se irán modificando y en dependencia del grado de tolerancia de estas especies a los cambios, su distribución podrá contraerse o expandirse a nuevas áreas. En la medida que en el futuro se obtengan registros de los loros dentro de la Península se podrán realizar nuevos modelos que permitan actualizar los rangos de distribución de estas especies, ya que los que se obtuvieron en este estudio solo dan una descripción temporal de sus patrones de distribución en la región.

AGRADECIMIENTOS. A J. del C. Puc, M. C. Aguirre, E. Victoria Chan, G. Quintal, M. L. Palomo y a todas las personas que brindaron sus servicios como guías en el trabajo de campo. Al personal de las áreas protegidas: Reservas de la Biosfera Sian Ka’an y Los Petenes, Parque Nacional Arrecifes de Cozumel, Fundación de Parques y Museos de Cozumel, Reserva de la Biosfera de los Petenes, Áreas de Protección de Flora y Fauna Otoch Ma’ax Yetel Kooh y Laguna de Términos. A los habitantes de los ejidos El Tablón, Arroyo Negro, Dos Lagunas, Divorciados, Pantera y Tigre Grande. A todos los que facilitaron registros de los loros y pericos en la Península de Yucatán: B. MacKinnon, A. Navarro-Sigüenza, C. Cáceres, J. Salgado, C. Macías y J. Vargas Soriano. A Y. Ferrer-Sánchez, O. R. Rojas Soto, E. Martínez Meyer, C. A. Ríos Muñoz, K. Renton, L. G. Esparza, D. Denis Ávila y A. T. Peterson por sus comentarios. Al CONACyT por el apoyo económico brindado a través de la beca 239499 y 21467. A los revisores del manuscrito por sus sugerencias y comentarios. A Idea Wild por los equipos donados.

\section{LITERATURA CITADA}

Anderson, R. P., Lew, D. \& Peterson, A. T. 2003. Evaluating predictive models of species' distributions: Criteria for selecting optimal models. Ecological Modelling, 162: 211-232.

Baldwin, R. A. 2009. Use of maximum entropy modeling in wildlife research. Entropy, 11: 854-866.

Blondel, J. 1969. Méthodes de dénombremente des populations d'ecologie. In: Lamotte, M. \& F. Bourliere (Eds.). Problemes d'ecologie: L'echantillonnage des peuplements animaux de milieux terres- 
tres. Masson, Paris, Francia. 304 pp.

Cantú-Guzmán, J. C., Sánchez-Saldaña, M. E., Grosselet, M. \& Silva-Gámez, J. 2007. Tráfico ilegal de pericos en México: una evaluación detallada. Defenders of Wildlife, Washington, D.C. $80 \mathrm{pp}$.

Collar, N. J., Gonzaga, L. P., Krabbe, N., Madroño Nieto, A., Naranjo, L. G., Parker, T. A. \& Wege, D. C. 1992. Threatened birds of the Americas: the ICBP/IUCN Red Data Book. International Council for Bird Preservation, Cambridge, U.K. 1150 pp.

CONAGUA. 2006. Estadísticas del Agua en México- SEMARNAT-CNA. 233 pp. <http://www.conagua.gob.mx/CONAGUA07/Publicaciones/Publicaciones/EAM_2006.pdf> (consultado 10 de septiembre de 2012).

Contreras-Medina, R., Luna-Vega, I. \& Ríos-Muñoz, C. A. 2010. Distribución de Taxus globosa (Taxaceae) en México: Modelos ecológicos de nicho, efectos del cambio del uso de suelo y conservación. Revista Chilena de Historia Natural, 83: 421-433.

Cuervo-Robayo, A. P., Téllez-Valdés, O., Gómez, M., Venegas-Barrera, C., Manjarrez, J. \& Martínez-Meyer, E. 2013. An update of high-resolution monthly climate surfaces for Mexico. International Journal of Climatology. <http://onlinelibrary.wiley.com/doi/10.1002/joc.3848/pdf> (Consultado 3 Noviembre 2013).

Davies, R. G, Orme, C. D. L., Storch, D., Olson, V. A., Thomas, G. H., Ross, S. G., Ding, T. S., Rasmussen, P. C., Bennett, P. M., Owens, I. P. F., Blackburn, T. M. \& Gaston, K. J. 2007. Topography, energy and the global distribution of bird species richness. Proceedings of the Royal Society B, 274: 1189-1197.

Dormann, C. F., Elith, J., Bacher, S., Buchmann, C., Carl, G., Carré, G., García-Marquéz, J. R., Gruber, B., Lafourcade, B., Leitão, P. J., Münkemüller, T., McClean, C., Osborne, P. E., Reineking, B., Schröder, B., Skidmore, A. K., Zurell, D. \& Lautenbach, S.. 2012. Collinearity: a review of methods to deal with it and a simulation study evaluating their performance. Ecography, 35: 001-020.

Elith, J. 2000. Quantitative methods for modeling species habitat: comparative performance and an application to Australian plants. Pp. 39-58. In: Fergurson, S. \& M. Burgman (Eds.). Quantitative methods for conservation biology. Springer, New York.

Elith, J. \& Graham, C. H. 2006. Novel methods improve prediction of species' distributions from occurrence data. Ecography, 29: 129-151.

ESRI. 1999. ArcView 3.2. ESRI (Environmental Scientific Research Institute). Redlands, California, USA.

Fielding, A. H. \& Bell, J. F. 1997. A review of methods for the assessment of prediction errors in conservation presence/absence models. Environmental Conservation, 24: 38-49.

Fischer, J., Lindenmayer, D. B., Nix, H. A., Stein, J. L. \& Stein, J. A. 2001. Climate and animal distribution: a climatic analysis of the Australian marsupial Trichosurus caninus. Journal of Biogeography, 28: 293-304.

Fitzpatrick, M. C., Gotelli, N. J. \& Ellison, A. M. 2013. MaxEnt versus MaxLike: empirical comparisons with ant species distributions. Ecosphere, 4: 55. <http://dx.doi.org/10.1890/ES13-00066.1> (consultado 10 de febrero de 2014).

Galparsoro, I., Borja, A., Bald, J., Liria, P. \& Chust, G. 2009. Predicting suitable habitat for the European lobster (Homarus gammarus), on the Basque continental shelf (Bay of Biscay), using Ecological-Niche Factor Analysis. Ecological Modeling, 220: 556-567.

Gómez-Orduño, J. O. 2006. Disponibilidad y ocupación de cavidades para tres especies de loros del género Amazona spp. en Calakmul, Campeche. Tesis de Maestría. El Colegio de la Frontera Sur. Campeche, México. 
Green, R. 2010. An overview of the effects of climate change on birds. BOU Proceedings - Climate Change and Birds. <http://www.bou.org.uk/bouproc-net/ccb/green.pdf> (consultado 6 de septiembre de 2011).

Harris, J. B. C., Dwi Putra, D., Gregory, S. D., Brook, B. W., Prawiradilaga, D. M., Sodhi, N. S., Wei, D. \& Fordham, D. A. 2014. Rapid deforestation threatens mid-elevational endemic birds but climate change is most important at higher elevations. Diversity and Distributions, 1-13. < http://onlinelibrary.wiley.com/doi/10.1111/ddi.12180/pdf> (consultado 7 de abril de 2014).

Hernandez, P. A., Graham, C. H., Master, L. L. \& Albert, D. L. 2006. The effect of sample size and species characteristics on performance of different species distribution modeling methods. Ecography, 29: 773-785.

Herrera-Herrera, J. R. 2001. Spatial and temporal distribution of the avifauna in the wetland and upland habitats of the Calakmul Region, in Mexico. Tesis de Doctorado. State University of New York. Syracuse, New York.

Hoenes, B. D. \& Bender, L. C. 2010. Relative habitat- and browse-use of native desert mule deer and exotic oryx in the greater San Andres Mountains, New Mexico. Human-Wildlife Interactions, 4: $12-24$.

Howell, S. N. G. \& Webb, S. 1995. A guide to the birds of Mexico and northern Central America. Oxford Univ. Press, London, UK. 868 pp.

Hutto, R. L., Letschet, S. M. P. \& Hendricks, P. 1986. A fixed-radius point count methods for nonbreeding and breeding season use. Auk, 103: 593 - 602.

Kreakie, B. J., Fan, Y. \& Keitt, T. H. 2012. Enhanced Migratory Waterfowl Distribution Modeling by Inclusion of Depth to Water Table Data. PLOS ONE, 7. <http://envsci.rutgers.edu/ yreinfelder/ Pub/2012PLoSone.pdf> (consultado 10 de septiembre de 2012).

Liu, C., Berry, P. M., Dawson, T. P. \& Pearson, R. G. 2005. Selecting thresholds of occurrence in the prediction of species distributions. Ecography, 28: 385-393.

Lugo-Hubp J., Acevedes-Quesada, J. F. \& Espinosa-Pereña, R. 1992. Rasgos geomorfológicos mayores de la Península de Yucatán. Revista Mexicana de Ciencias Geológicas, 10: 143-150.

Macías-Caballero, C., \& Iñigo-Elías, E. E. 2003. Evaluación del estado de conservación actual de las poblaciones de Loro cabeza amarilla (Amazona oratrix) en México. Informe Final, Proyecto AS002, Comisión Nacional para el uso y conocimiento de la Biodiversidad, México, México.

MacKinnon H., B. 2005. Birds of Yucatan Peninsula. Amigos de Sian Ka’an, AC. Cancún, Quintana Roo, México.

Márdero, S., Nickl, E., Schmook, B., Schneider, L., Rogan, J., Christman, Z. \& Lawrence, D. 2012. Sequías en el sur de la Península de Yucatán: análisis de la variabilidad anual y estacional de la precipitación. Investigaciones Geográficas, 78: 19-33.

Marini, M. Â., Barbet-Massin, M., Lopes, L. E. \& Jiguet, F. 2010. Predicting the occurrence of rare Brazilian birds with species distribution models. Journal of Ornithology, 151: 857-866.

Marmion, M., Parviainen, M., Luoto, M., Heikkinen, R. K. \& Thuilier, W. 2009. Evaluation of consensus methods in predictive species distribution modelling. Diversity and Distributions, 15: 59-69.

Martínez, E. \& Galindo-Leal, C. 2002. La vegetación de Calakmul, Campeche, México: clasificación, descripción y distribución. Boletín de la Sociedad Botánica de México, 71: 7-32.

Mateo, R. G, Felicísimo, A. M. \& Muñoz, J. 2010. Effects of the number of presences on the reliability and stability of MARS species distribution models: The importance of regional niche variation and ecological heterogeneity. Journal of Vegetation Science, 21: 908-922.

Mateo, R. G., Felicísimo, A. M. \& Muñoz, J. 2011. Modelos de distribución de especies: Una revisión sintética. Revista Chilena de Historia Natural, 84: 217-240. 
Mateo, R. G., de la Estrella, M., Felicísimo, A. M., Muñoz, J. \& Guisan, A. 2013. A new spin on a compositionalist predictive modelling framework for conservation planning: A tropical case study in Ecuador. Biological Conservation, 160: 150-161.

Monterrubio-Rico, T. C., Renton, K., Ortega-Rodríguez, J. M., Pérez-Arteaga, A. \& CancinoMurillo, R. 2010. The Endangered yellow-headed parrot Amazona oratrix along the Pacific coast of Mexico. Oryx, 44: 602-609.

Monterrubio-Rico, T. C., De Labra Hernández, M. A., Ortega-Rodríguez, J. M., Cancino-Murillo, R. \& Villaseñor-Gómez, J. F. 2011. Distribución actual y potencial de la guacamaya verde en Michoacán, México. Revista Mexicana de Biodiversidad, 82: 1311-1319.

Mota-Vargas, C., Rojas-Soto, O. R., Lara, C., Castillo-Guevara, C. \& Ballesteros-Barrera, C. 2013. Geographic and ecological analysis of the Bearded Wood Partridge Dendrortyx barbatus: some insights on its conservation status. Bird Conservation International, 23: 371-385.

Navarro-Sigüenza, A. G., Peterson, A. T. \& Gordillo-Martínez, A. 2003. Museums working together: the atlas of the birds of Mexico. Pp. 207-225. In: Collar, N., C. Fisher \& C. Feare (Eds.). Why museums matter: avian archives in an age of extinction. Bulletin British Ornithologists' Club Supplement, 123A.

Navarro, A. G. \& Peterson, A. T. 2007. Amazona oratrix (loro cabeza-amarilla) residencia permanente. Distribución potencial. Extraído del proyecto CE015: Mapas de las aves de México basados. Museo de Zoología, Facultad de Ciencias, UNAM \& University of Kansas, Museum of Natural History. Financiado por la Comisión Nacional para el Conocimiento y Uso de la Biodiversidad (CONABIO). México. <http://www.conabio.gob.mx/informacion/gis/layouts/amaz_oratgw.gif> (consultado 17 de octubre de 2013).

Orellana-Lanza, R., Espadas-Manrique, C., Conde-Álvarez, C. \& Gay-García, C. 2009. Atlas escenarios de cambio climático en la Península de Yucatán. Centro de Investigación Científica de Yucatán, A.C. Mérida, Yucatán, México. <http://www.cambioclimatico.yucatan.gob.mx/atlas-cambio-climatico/index.php> (consultado 2 de octubre de 2012).

Pearson, R. G., Raxworthy, C. J., Nakamura, M. \& Peterson, T. A. 2007. Predicting species distributions from small numbers of occurrence records: a test case using cryptic geckos in Madagascar. Journal of Biogeography, 34: 102-117.

Pérez-García, B. \& Liria, J. 2013. Modelos de nicho ecológico fundamental para especies del género Thraulodes (Ephemeroptera: Leptophlebiidae: Atalophlebiinae). Revista Mexicana de Biodiversidad, 84: 600-611.

Peterson, A. T., Ortega-Huerta, M. A., Bartley, J., Sánchez-Cordero, V., Soberón, J., Buddemeier, R. H. \& Stockwell, D. R. B. 2002. Future projections for Mexican faunas under global climate change scenarios. Nature, 416: 626-629.

Peterson, A. T., Soberón, J., Pearson, R. G., Anderson, R. P., Martínez-Meyer, E., Nakamura, M. \& Araujo, M. B. 2011. Ecological niches and geographic distributions. Princeton University Press, Princeton. 328 pp.

Phillips, S. J., Anderson, R. P. \& Schapire, R. E. 2006. Maximum entropy modeling of species geographic distributions. Ecological Modelling, 190: 231-259.

Phillips, S. J. \& Dudík, M. 2008. Modeling of species distribution with MaxEnt: new extensions and a comprehensive evaluation. Ecography, 31: 161-175.

Phillips, S. J., Dudík, M., Elith, J., Graham, C. H., Lehmann, A., Leathwick, J. \& Ferrier, S. 2009. Sample selection bias and presence-only distribution models: implications for background and pseudo-absence data. Ecological Applications, 19: 181-197.

Puc-Cabrera, J. C. 2008. Importancia de las aguadas para los ensambles de aves en el sureste de Campeche. Tesis de Licenciatura. Universidad Autónoma de Campeche. Campeche, México. 
Plasencia-Vázquez et al.: Distribución potencial de psitácidos neotropicales

Rahbek, C. 2005. The role of spatial scale and the perception of large-scale species-richness patterns. Ecology Letters, 8: 224-239.

Renton, K, \& Salinas-Melgoza, A. 2002. Amazona finschi (Sclater 1864) Loro corona lila. Pp. 341342. In: Noguera, F. A., J. H. Vega-Rivera \& A. N. García-Aldrete, M. Quesada (Eds.). Historia Natural de Chamela. Instituto de Biología, Universidad Nacional Autónoma de México. Distrito Federal, México.

Renton, K. \& Salinas-Melgoza, A. 2004. Climatic variability, nest predation, and reproductive output of lilac-crowned parrots (Amazona finschi) in tropical dry forest of western Mexico. Auk, 121: 12141225.

Renton, K. 2009. Lilac-crowned Parrot (Amazona finschi). Neotropical Birds Online (T. S. Schulenberg, Editor). Ithaca: Cornell Lab of Ornithology; retrieved from Neotropical Birds Online: <http:// neotropical.birds.cornell.edu/portal/species/overview?p_p_spp=23510> (consultado 29 de febrero de 2012).

Ríos-Muñoz, C. A. 2002. Caracterización geográfica de la familia psittacidae (aves) utilizando un modelo predictivo. Tesis de licenciatura. Departamento de Biología, Facultad de Ciencias, Universidad Nacional Autónoma de México. México D.F.

Ríos-Muñoz, C. A. \& Navarro-Sigüenza, A. G. 2009. Efectos del cambio de uso de suelo en la disponibilidad hipotética de hábitat para los psitácidos de México. Ornitología Neotropical, 20: 491509.

Rojas-Soto, O. R., Sosa, V., Ornelas, J. F. 2012. Forecasting cloud forest in eastern and southern Mexico: conservation insights under future climate change scenarios. Biodiversity and Conservation, 21: 2671-2690.

Royle, J. A., Chandler, R. B., Yackulic, C. \& Nichols, J. D. 2012. Likelihood analysis of species occurrence probability from presence-only data for modelling species distributions. Methods in Ecology and Evolution, 3: 545-554.

Salinas-Melgoza, A. \& Renton, K. 2005. Seasonal variation in activity patterns of juvenile lilac-crowned parrots in tropical dry forest. Wilson Bulletin, 117: 291-295.

SEMARNAT. Secretaría de Medio Ambiente y Recursos Naturales. 2010. Norma Oficial Mexicana NOM-059-SEMARNAT-2010. Diario Oficial de la Federación (DOF).

Svoray, T. \& Karnieli, A. 2010. Rainfall,topography and primary production relationships in a semiarid ecosystem. Ecohydrology, 4: 56-66.

Venier, L. A., Pearce, J., McKee, J. E., McKenney, D. W. \& Niemi, G. J. 2004. Climate and satellite-derived land cover for predicting breeding bird distribution in the Great Lakes Basin. Journal of Biogeography, 31: 315-331.

Villers, L., Arizpe, N., Orellana, R., Conde, C. \& Hernández, J. 2009. Impactos del cambio climático en la floración y desarrollo del fruto del café en Veracruz, México. Interciencia, 34: 322-329.

Warren, D. L. \& Seifert, S. N. 2011. Ecological niche modeling in MaxEnt: the importance of model complexity and the performance of model selection criteria. Ecological Applications, 21: 335-342.

White, D. A \& Darwin, S. P. 1995. Woody vegetation of tropical lowland deciduous forests and Mayan ruins in the north-central Yucatan Peninsula, Mexico. Tulane Studies in Zoology and Botany, 30: $1-25$.

Wright, S. J., Carrasco, C., Calderón, O. \& Paton, S. 1999. The El Niño southern oscillation, variable fruit production, and famine in a tropical forest. Ecology, 805: 1632-1647.

Yackulic, C. B., Chandler, R., Zipkin, E. F., Royle, J. A., Nichols, J. D., Campbell Grant, E. H. \& Veran, S. 2013. Presence-only modelling using MAXENT: when can we trust the inferences? Methods in Ecology and Evolution, 4: 236-243. 